\title{
A Novel Technique to Prepare Cast Al-bearing alloy/Wrought Steel Bimetallic Specimen for Interfacial Shear Strength
}

\author{
M. Ramadan, T. Subhani, W. Rajhi, B. Ayadi, A. S. Al-Ghamdi
}

\begin{abstract}
A novel mold was designed and manufactured for facile preparation of cast/wrought bimetallic specimen for subsequent mechanical performance. To ensure the easy manufacturing of bimetallic specimen, an aluminum bearing alloy was cast on a wrought carbon steel substrate after tinning process for adequate interfacial bonding in the especially prepared mold, which was characterized mechanically by lap-joint shear test to measure bonding strength between the two materials and by microhardness test for hardness profile across the interface. Optical and electron microscopy was employed for the microstructural observation of the interface to relate it with the mechanical performance of bimetallic material for bearing and automotive applications while electron dispersive spectroscopy mapped the elemental distribution across interface.
\end{abstract}

Keywords: Interfacial, Shear strength, Bimetallic, Bearing materials.

\section{INTRODUCTION}

Bimetallic materials constitute a special group of materials containing two materials bonded together by appropriate adhesion techniques. Bearing material is a typical example of bimetallic materials, whereas automobile industry is also demanding lightweight bimetallic materials for improved fuel economy [1, 2]. In bimetallic materials, the bonding materials may be metals, polymers, ceramics or composites. Among metallic materials, tin and lead-based alloys (also called as white metals), bronzes and aluminum alloys are common while plastics and rubbers are the examples of polymers. Amongst ceramics, carbides and oxides are the typical examples especially glasses while carbon and glass fiber reinforced polymeric matrix composites are the examples of composite materials.

Revised Manuscript Received on February 05, 2020.

* Correspondence Author

Mohamed Ramadan, Department of Mechanical Engineering, College of Engineering, University of Ha'il, Saudi Arabia. Central Metallurgical Research and Development Institute, Cairo, Egypt. mrnais3@yahoo.com

Tayyab Subhani*, Department of Mechanical Engineering, College of Engineering, University of Ha'il, Saudi Arabia. drtayyabsubhani@gmail.com

Wajdi Rajhi, Department of Mechanical Engineering, College of Engineering, University of Ha'il, Saudi Arabia. w.rajhi@uoh.edu.sa

Badreddine Ayadi, Department of Mechanical Engineering, College of Engineering, University of Ha'il, Saudi Arabia. b.ayadi@uoh.edu.sa

Abdulaziz Salem Al-Ghamdi, Department of Mechanical Engineering, College of Engineering, University of Ha'il, Saudi Arabia. a.alghamdi @uoh.edu.sa

(c) The Authors. Published by Blue Eyes Intelligence Engineering and Sciences Publication (BEIESP). This is an open access article under the CC BY-NC-ND license (http://creativecommons.org/licenses/by-nc-nd/4.0/)
In bimetallic materials, the depositing metal can be attached to substrate surface either chemically or mechanically. The two common techniques are casting and welding wherein bearing material is deposited on substrate in liquid state and is solidified subsequently without further strengthening by cold working. Two casting processes, i.e. centrifugal and static casting are used. For cylindrical parts, centrifugal casting process is used wherein centrifugal force is the cause of adhesion between the cast materials and substrate. On the other hand, conventional static casting is considered as the standard option for simple configuration of substrate such as flat surface [3]. As a third option, thermal spraying process is used to deposit cast alloy on complicated substrate surfaces. Diffusion bonding is yet another technique to prepare bimetallic materials.

A number of variables such as load, temperature and contact pressure define the selection of an appropriate bimetallic material for a specific application. Together with desired hardness, compressive strength, impact resistance and fatigue properties, the depositing material must be strongly adhered to substrate material [4].

Among a wide range of available depositing materials, aluminum alloys have the advantage of high specific properties. Another edge of aluminum alloys is their high thermal conductivity. The common alloying elements in aluminum alloys are silicon, cadmium, nickel, copper and tin. The tin loadings of up to $30 \%$ are used for special aluminum material possessing the characteristics of heavy loading and high speed. The addition of copper improves the hardness of aluminum bearing materials. Usually aluminum is used with steel backing and the bonding strength between the aluminum and steel is critical. The combination of steel with aluminum alloys is also under high demand in automobile industry [5] due to the fact that specific mechanical performance of aluminum are combined with the high mechanical properties of steels to resolve the issue between safety and fuel economy [6].

The bonding strength developed due to the evolution of microstructure at the interface is the prime requisite [7]. The bonding strength depends on a variety of variables including the surface roughness of substrate, cleaning and tinning/zinc coating of substrate, solidification rate of pouring cast alloy, addition of alloying elements in cast alloy and temperature of substrate [8]. For cylindrical shapes of lining, Chalmers test is applied to evaluate the bonding strength [9] while there is a need to estimate the lining of flat surfaces. Also Chalmers test is difficult to operate and hence there is a need to devise a novel and facile technique to appraise the bonding strength of bearing material lining. 


\section{A Novel Technique to Prepare Cast Al-bearing alloy/Wrought Steel Bimetallic Specimen for Interfacial Shear Strength}

In this study, a bimetallic cast aluminum/wrought steel material is prepared by a novel specimen-preparing technique for subsequent measurement of interfacial shearing strength. Aluminum alloy was bonded with substrate carbon steel by pouring the molten aluminum alloy through static or gravity casting. The prepared sample was removed from the mold and tested for shear strength after machining down the thickness of aluminum to match steel strip.

\section{EXPERIMENTAL}

\section{A. Materials and Manufacturing}

Bimetallic Al-Sn-Si-Cu/Steel specimen was prepared by depositing liquid Al-12Sn-4Si-1Cu alloy on solid low carbon steel in a custom-made mold. The mold was especially designed to prepare bimetallic specimens for shear testing comprising a cast material bonded with a wrought material. A tinning process was used to improve the adhesion quality between the two materials because it is difficult to trigger the reaction between aluminum and steel [10-12].

For tinning process, a flux was prepared containing $24 \mathrm{~g}$ zinc chloride, $6 \mathrm{~g}$ sodium chloride, $3 g$ ammonium chloride, $1 \mathrm{ml}$ hydrochloric acid and $1 \mathrm{ml}$ water. $10 \mathrm{~g}$ of the prepared flux was mixed with $1 \mathrm{~g}$ of pure $\mathrm{Sn}$ powder of grain size $48 \mu \mathrm{m}$; the mixture was distributed on the freshly ground surface of wrought carbon steel strip of dimensions $20 x 20 \mathrm{~mm}$ with SiC emery paper of grit size 400 , which was followed by heating the strip on hot plate at $350^{\circ} \mathrm{C}$ for $2 \mathrm{~min}$. $\mathrm{Sn}$ in the mixture settled down while flux appeared to the surface. After tinning process, the carbon steel substrate was cooled to room temperature. Subsequently, the specimen was washed with warm water and the flux was removed from the surface of carbon steel substrate using loose cotton soaked with warm water.

Aluminum alloy of the composition Al-12Sn-4Si-1Cu was made and deposited on AISI-1015 carbon steel containing $0.15 \%$ carbon using casting process to prepare bimetallic specimen (Table 1). Al-12Sn-4Si-1Cu alloy was made using a bar of Al-25\%Si master alloy, pure $\mathrm{Sn}$, pure $\mathrm{Cu}$ and pure Al. The alloy was melted in a graphite crucible using an electrical furnace at a temperature of $750^{\circ} \mathrm{C}$ for $30 \mathrm{~min}$. At a temperature of $720^{\circ} \mathrm{C}$, the prepared alloy was poured into the especially designed mold upon the tinned carbon steel substrate, which was heated at $350^{\circ} \mathrm{C}$.

\section{B. Characterization}

After casting and solidification of Al-Sn-Si-Cu alloy on carbon steel substrate using the specially designed mold, the bimetallic specimen was removed from the mold and the thickness of the cast Al-Sn-Si-Cu alloy was reduced to $2 \mathrm{~mm}$ to match the thickness of counter carbon steel strip. The bimetallic specimen was then ready for shear testing and microstructural observation. The bonded area between cast Al-Sn-Si-Cu alloy and wrought carbon steel was $20 \times 20 \mathrm{~mm}=400 \mathrm{~mm}^{2}$ thus making a lap joint between the two materials.

For shear testing, the bimetallic specimen was placed in the grips of universal tensile testing machine Instron 5969 characterized by the maximum load of $50 \mathrm{KN}$. The shear test was carried out at a constant strain rate of $1 \mathrm{~mm} / \mathrm{min}$ until the failure of the specimen. At least five specimens were tested to obtain the average value and confirm the auditability of the preparation of specimens using the novel mold.
For microstructural observation, standard metallographic practice was adopted comprising grinding and polishing operations followed by etching. For etching, a solution of $0.5 \mathrm{ml}$ nitric acid, $0.3 \mathrm{ml}$ hydrochloric acid, $0.2 \mathrm{ml}$ hydrofluoric acid and $19 \mathrm{ml}$ water was used. To acquire microstructures, Olympus optical microscope (IMM-901, Metkon Instruments, Japan) fitted with digital camera was used for optical microscopy. The electron microscopy of the specimen was performed on scanning electron microscope (SEM) TESCAN, MIRA-III, FEG-SEM, Czech Republic attached with electron dispersive spectroscopy (EDS). The images were acquired in secondary electron imaging mode at an accelerating voltage of $10 \mathrm{kV}$. The hardness testing was carried out at the load of $0.25 \mathrm{kgf}$ for the dwell time of $5 \mathrm{sec}$.

\section{RESULTS AND DISCUSSION}

Figure 1a shows the three-dimensional image of the especially designed mold to prepare cast/wrought bimetallic specimen while Figure $1 \mathrm{~b}$ depicts the drawing of the mold showing three different views. A strip of substrate steel of width $20 \mathrm{~mm}$ and thickness $2 \mathrm{~mm}$ can be inserted into the mold through the opening at the back. The length of steel strip of at least $60 \mathrm{~mm}$ can be accommodated in the mold. After inserting the steel strip in the mold, a cavity of length $60 \mathrm{~mm}$, width $20 \mathrm{~mm}$ and height $8 \mathrm{~mm}$ forms, which serves as the pool for pouring the cast materials.
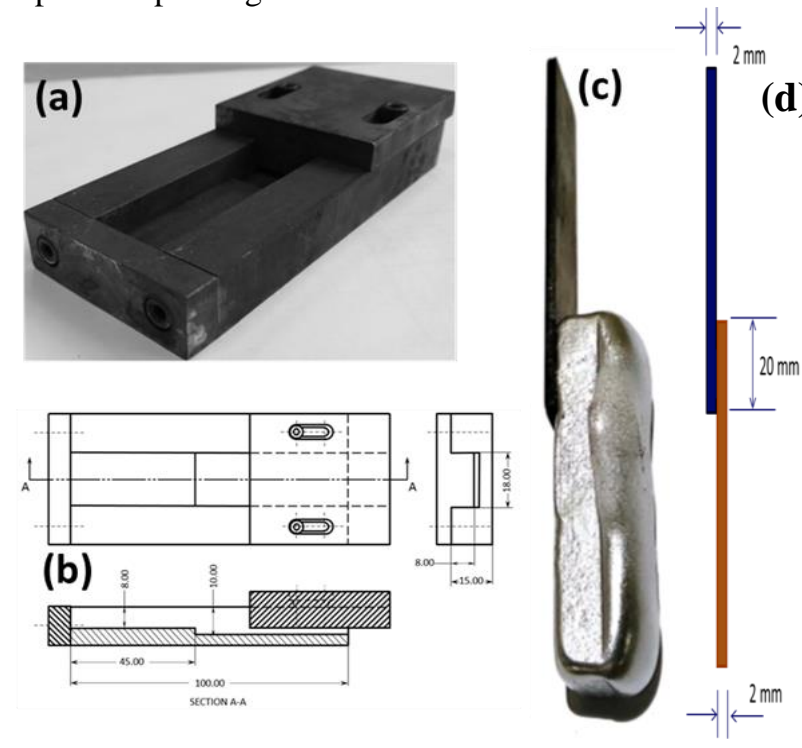

Fig. 1. Shear test (a) mold and (b) diagram for preparing bimetallic specimens. Bimetallic Al-Sn-Si-Cu alloy/carbon steel specimen after pouring Al-Sn-Si-Cu alloy pouring on substrate carbon steel (c) before and (d) after machining excessive Al-Sn-Si-Cu alloy.

Figure 1c shows the as-prepared bimetallic Al-Sn-Si-Cu alloy/carbon steel specimen, which was removed from the mold after pouring, solidification and cooling of Al-Sn-Si-Cu alloy. After removing the additional thickness from the cast alloy, it was matched with its counterpart of steel of $2 \mathrm{~mm}$; Figure 1d shows the specimen after machining ready for shear testing. 
Figure 2a is the image of bimetallic specimen held in the grips of the universal testing machine for shear test. After shear testing, the broken specimen is shown in Figure 2b; it can be seen that after debonding the specimen, Sn was still attached to the surface of steel strip,

which not only indicates the appropriateness of tinning process used in the present work to improve the bonding quality but it also reveals a good interfacial bond between the cast aluminum and wrought steel materials, as also observed in the magnified image in Figure 2c.

Stress-strain diagram of a mechanical test is always a good indicator of a successful characterization. The shear stress-strain diagram in Figure 2d shows a smooth and linear rise in the stress together with the increase in the strain within the specimen. Reliable interfacial shear strength of $\sim 6 \mathrm{MPa}$ was achieved, which indicates a good bonding between the two materials. Indeed, the obtained shear strength value can be increased by changing the variables such as the roughness of the adjoining surfaces, adjustment of alloying elements in the cast aluminum alloy, tinning process improvement.
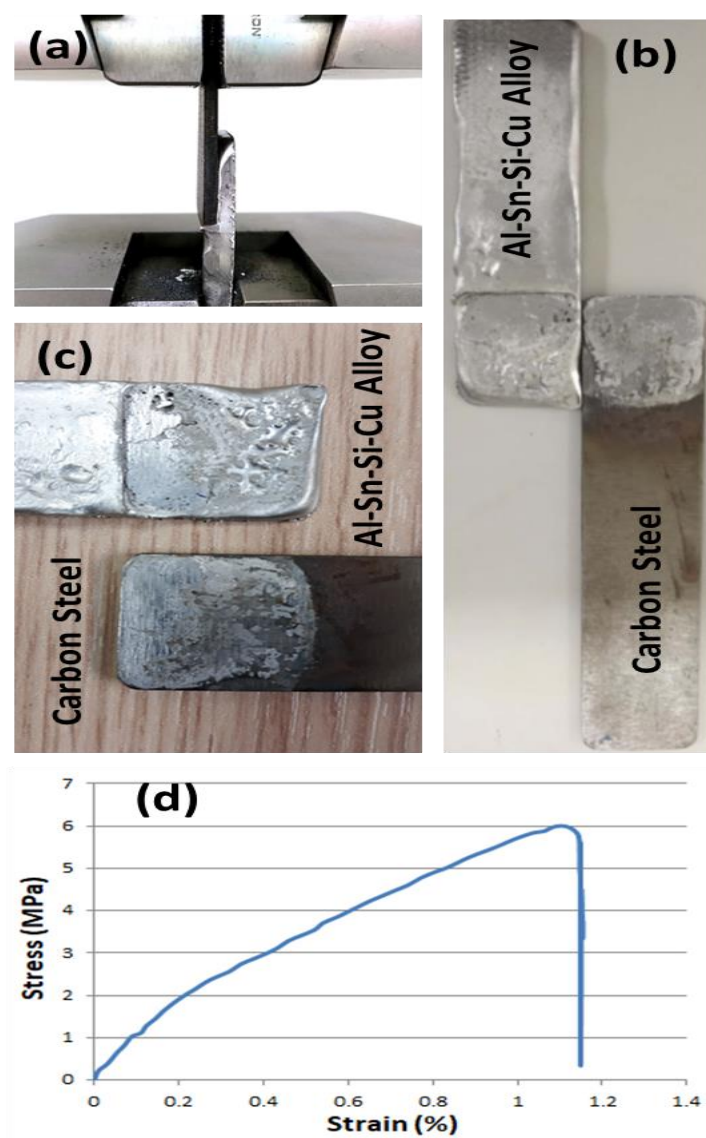

Fig. 2. Shear test specimen of Al-Sn-Si-Cu alloy/carbon steel bimetallic material: (a) before and (b) after testing along with (c) magnified image; (d) stress-strain diagram of shear test.

Figure 3 shows the microstructures of materials in bimetallic strip and the interface. Figure 3a shows the image of Al-Sn-Si-Cu alloy showing the $\alpha$-Al matrix and different phases of $\mathrm{Sn}$ and $\mathrm{Si}$ on grain boundaries of $\alpha$-Al grains while the Figure $3 c$ shows the typical steel microstructure containing ferrite and pearlite in proportion to the carbon content in the steel. The interfacial region, as shown in Figure 3b contains a thin layer of Sn that was deposited on solid carbon steel substrate surface before performing tinning process.

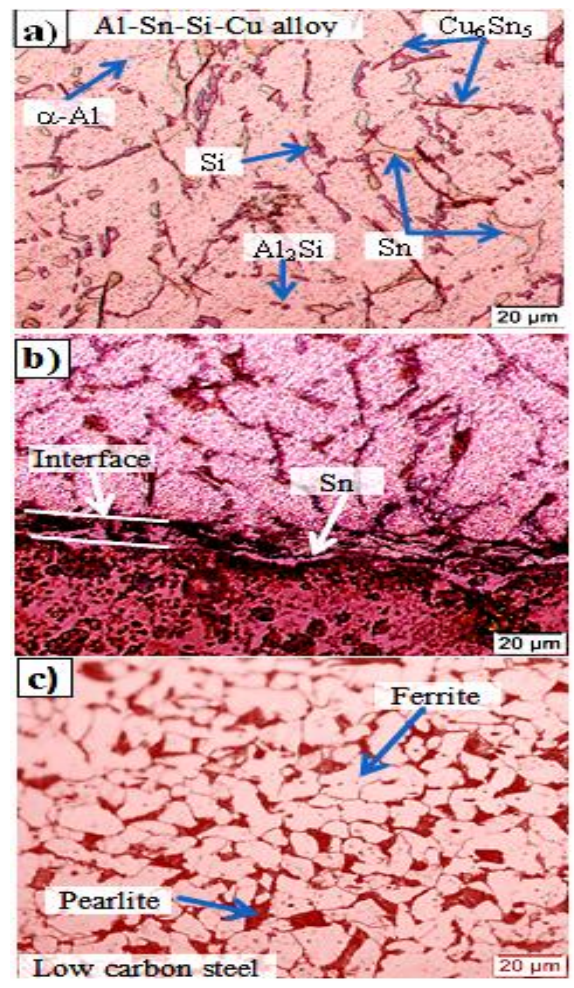

Fig. 3. Microstructures of Al-Sn-Sin-Cu/Carbon steel bimetal: (a) Al-Sn-Si-Cu alloy region, (b) interfacial region and (c) Carbon steel substrate

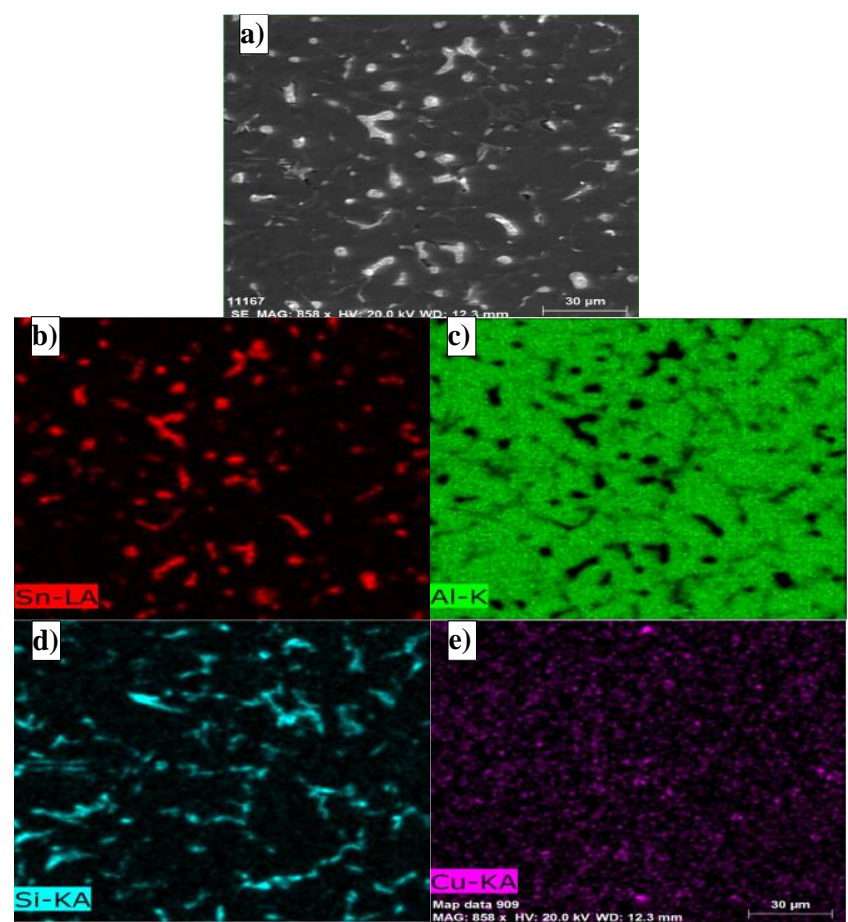

Fig. 4. SEM image of aluminum alloy Al-Sn-Sin-Cu in bimetallic material (a) and EDS mapping for the elemental analysis of (b) Sn, (c) Al, (d) Si and (e) Cu.

Figure 4 a shows SEM image of aluminum alloy $\mathrm{Al}-\mathrm{Sn}-\mathrm{Sin}-\mathrm{Cu}$, which was cast upon steel substrate to prepare bimetallic material. The cast structure can be seen together with the observation of porosity. The EDS mapping for the elemental distribution of tin, aluminum, silicon and copper is shown in Figures 4 b, c, d,

Published By:

Blue Eyes Intelligence Engineering DOI: 10.35940/ijeat.C6084.029320

\& Sciences Publication

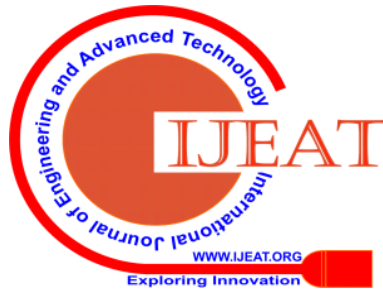




\section{A Novel Technique to Prepare Cast Al-bearing alloy/Wrought Steel Bimetallic Specimen for Interfacial Shear Strength}

and e. A uniform distribution of elements, i.e. tin, silicon and copper was witnessed, which evidently showed the homogeneous distribution of elements during the solidification of aluminum alloy without segregation.

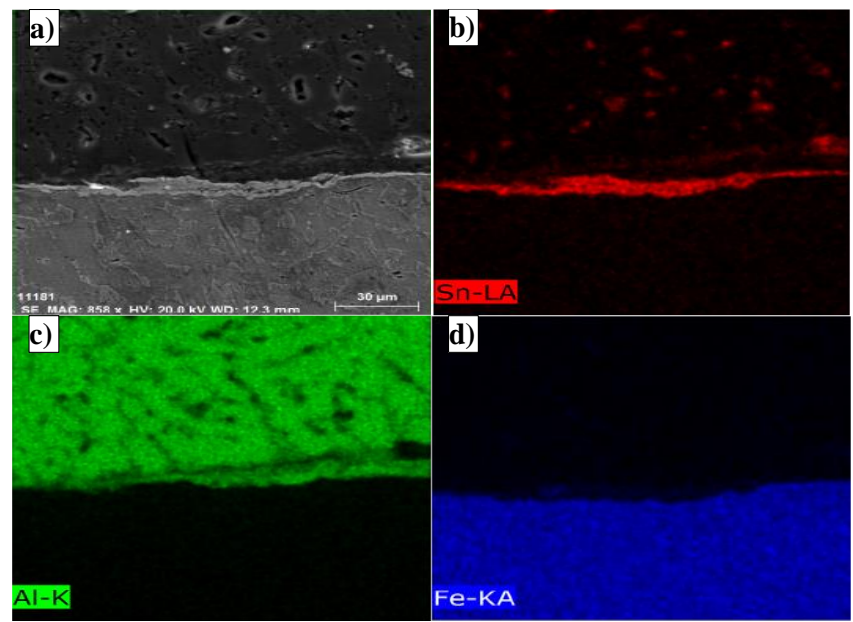

Fig. 5. SEM image of the interface of bimetallic material (a) and EDS mapping for the elemental analysis of (b) Sn, (c) Al and (d) Fe.

Figure 5 a shows the SEM image of the interface of bimetallic material. (a) and EDS mapping for the elemental analysis of (b) Sn, (c) Al and (d) Fe. A good adhesion between the aluminum alloy and steel can be observed without the indication of defects at the interface. The distribution of tin in aluminum alloy can be seen in Figure $5 \mathrm{~b}$ along with the interlayer of tin along the interface due to tinning process. Figures $5 \mathrm{c}$ and $\mathrm{d}$ are the EDS images of aluminum and iron in aluminum alloys and steel, respectively.

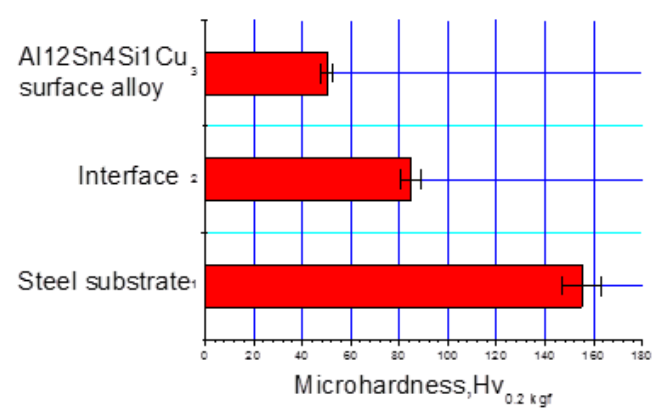

Fig. 6. Microhardness values of the interface of bimetallic material, and joining materials of (b) aluminum alloy Al12Sn4SiCu and (c) substrate steel.

The microhardness values of the interface and the adjoining materials in bimetallic material are shown in Figure 6. Aluminum alloy has shown the minimum hardness value of $50 \pm 2 \mathrm{HV}$ while the steel has the maximum hardness of $156 \pm 7 \mathrm{HV}$; the interface revealed an intermediate value of $93 \pm 4 \mathrm{HV}$.

In short, the combination of tinning process with the technique of pouring of cast metal upon a solid substrate material in especially designed mold offers an easy route of testing the shear strength of a variety of bimetallic materials containing cast and wrought parts so as to obtain the optimum combination of bimetallic material for bearing and automobile applications.
Table:1 Chemical compositions of aluminum alloy and low carbon steel used for bimetallic specimen fabrication

\begin{tabular}{|c|c|c|c|c|c|c|c|c|}
\hline $\begin{array}{c}\text { Bimetallic } \\
\text { Material (\%) }\end{array}$ & C & Si & Mn & Cu & Sn & Cr & Al & Fe \\
\hline $\begin{array}{c}\text { Al12Sn4SiCu } \\
\text { Alloy }\end{array}$ & - & 4 & - & 1 & 12 & - & Bal & - \\
\hline Carbon Steel & 0.15 & 0.30 & 0.48 & 0.20 & - & 0.14 & - & Bal \\
\hline
\end{tabular}

\section{CONCLUSIONS}

Bimetallic specimen was prepared using a novel mold, which was especially designed and fabricated for facile manufacturing of bimetallic specimens for succeeding characterization of interfacial strength through shear test. To confirm the aptness of the designed mold, Al-Sn-Si-Cu bearing alloy was cast on wrought AISI-1015 carbon steel substrate after tinning process for adequate bonding. The prepared specimen was tested mechanically for lap-joint shear test for reliable interfacial bonding strength between the two materials and hardness test for microhardness profile across interface, which was substantiated by the microscopic observation through electron and optical microscopy. The present technique offers a convenient approach for preparing bimetallic specimens for subsequent mechanical performance for a variety of combinations of cast and wrought materials to address the rising demand in bearing and automotive applications.

\section{ACKNOWLEDGMENT}

The present research work has been undertaken within the funded research projects of University of Hail, Kingdom of Saudi Arabia (Deanship of Scientific Research RG-191193). This is gratefully acknowledged.

\section{REFERENCES}

1. J. Shin, T. Kim, K. Lim, H. Cho, D. Yang, C. Jeong, S. Yi, Effects of steel type and sandblasting pretreatment on the solid-liquid compound casting characteristics of zinc-coated steel/aluminum bimetals, Journal of Alloys and Compounds 778 (2019) 170-185.

2. M. Ramadan, N. Fathy, K. Abdel-Halim, A. Alghamdi, New trends and advances in bi-metal casting technologies, International Journal of ADVANCED AND APPLIED SCIENCES 6 (2019) 75-80.

3. M. Ramadan, K.M. Hafez, K.S. Abdel Halim, N. Fathy, T. Chiba, H Sato, Y. Watanabe, Influence of Heat Treatment on Interface Structure of Stainless Steel/Gray Iron Bimetallic Layered Castings, Applied Mechanics and Materials 873 (2017) 3-8.

4. M. Ramadan, Interface Structure and Elements Diffusion of As-Cast and Annealed Ductile Iron/Stainless Steel Bimetal Castings, Engineering, Technology and Applied Science Research 8 (2018).

5. W. Jiang, Z. Fan, C. Li, Improved steel/aluminum bonding in bimetallic castings by a compound casting process, Journal of Materials Processing Technology 226 (2015) 25-31.

6. J. Shin, T. Kim, D. Kim, D. Kim, K. Kim, Castability and mechanical properties of new 7xxx aluminum alloys for automotive chassis/body applications, Journal of Alloys and Compounds 698 (2017) 577-590.

7. L. Shao, Y. Shi, J.K. Huang, S.J. Wu, Effect of joining parameters on microstructure of dissimilar metal joints between aluminum and galvanized steel, Materials \& Design 66 (2015) 453-458.

8. W. Jiang, Z. Fan, G. Li, C. Li, Effects of zinc coating on interfacia microstructures and mechanical properties of aluminum/steel bimetallic composites, Journal of Alloys and Compounds 678 (2016) 249-257.

9. P. Diouf, A. Jones, Investigation of Bond Strength in Centrifugal Lining of Babbitt on Cast Iron, Metallurgical and Materials Transactions A 41(3) (2010) 603-609. 
10. Y. Liu, X. Bian, K. Zhang, C. Yang, L. Feng, H.S. Kim, J. Guo, Interfacial microstructures and properties of aluminum alloys/galvanized low-carbon steel under high-pressure torsion, Materials \& Design 64 (2014) 287-293.

11. J.C. Viala, M. Peronnet, F. Barbeau, F. Bosselet, J. Bouix, Interface chemistry in aluminium alloy castings reinforced with iron base inserts, Composites Part A: Applied Science and Manufacturing 33(10) (2002) 1417-1420.

12. A. Bouayad, C. Gerometta, A. Belkebir, A. Ambari, Kinetic interactions between solid iron and molten aluminium, Materials Science and Engineering: A 363(1) (2003) 53-61.

\section{AUTHORS PROFILE}

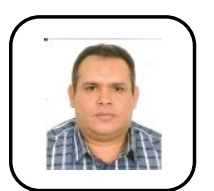

Prof. Dr. Mohamed Ramadan is currently working as Professor in the Department of Mechanical Engineering, College of Engineering, University of Ha'il, Saudi Arabia, and also as Research Associate Professor in Central Metallurgical Research and Development Institute, Cairo, Egypt. He received his B.Sc in Metallurgical Engineering from Suez Canal University, Faculty of Petroleum and Mining Engineering, Metallurgical Engineering dept. in Egypt, M.Sc. in Metallurgical Engineering from Cairo University and $\mathrm{PhD}$ in Materials Processing Engineering from Department of Materials, Physics and Energy Engineering, Nagoya University, Japan. Before joining University of Hail, he worked as researcher at Central Metallurgical Research and Development Institute, CMRDI, Manufacturing Technology, Casting Technology Department in Egypt. Over the years, He has been interested in metallurgical and materials engineering of metals, alloys, bimetal casting, and metal matrix composites. He published more than 60 scientific research papers in international journals and conferences in the field of metallurgical and materials engineering. He is the reviewers of several international journals and the member of different national and international materials organizations.

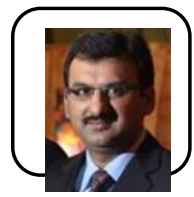

Dr. Tayyab Subhani is Assistant Professor of Materials Science and Engineering in the Department of Mechanical Engineering, College of Engineering, University of Ha'il, Saudi Arabia. He has 20 years of teaching, research, management and industrial experience. He is a graduate from Imperial College London, UK, where he performed his $\mathrm{PhD}$ research work in nanocomposites. He has more than 70 publications. His area of research is Nanotechnology, Nanomaterials, Nanocomposites, Advanced Materials, Manufacturing and Characterization. He has served as Head Graduate Program Office in the Institute of Space Technology, Pakistan. He has undertaken many projects funded by Research and Development Organizations. He has supervised many Bachelor, Master and Doctorate students. He is the reviewers of several international journals and the member of different national and international materials organizations.

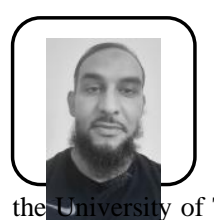

Dr. Wajdi Rajhi is currently working as Assistant Professor in the Department of Mechanical Engineering, College of Engineering, University of Ha'il, Saudi Arabia. He has 10 years of teaching, research and industrial experience. He earned two $\mathrm{PhD}$ degrees in Mechanical Engineering in 2014. The first one was from of Technology of Troyes (UTT), France and the second one from the Higher National Engineering School of Tunis (ENSIT), Tunisia. His area of research is Mechanics of materials and structures especially the damage of materials during manufacturing and materials processing.

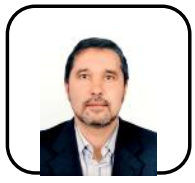

Dr. Badreddine Ayadi is currently working as Assistant Professor in the Department of Mechanical Engineering, College of Engineering, University of Ha'il, Saudi Arabia. He has 27 years of teaching, research, management and industrial experience. His area of research is Manufacturing. He has supervised many Bachelor and Master students.

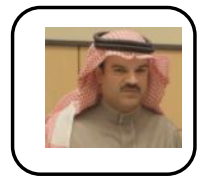

Dr. Abdulaziz Alghamdi is an associate professor of Materials Science \& Engineering in the Department of Mechanical Engineering, College of Engineering, University of Ha'il, Saudi Arabia. Dr. Alghamdi was the chairman of the mechanical engineering department at the University of Hail in 2014, then vice dean for academic affairs in 2015. In 2016, he was appointed as the dean of college of engineering. In addition to his membership of various standing committees and councils in the University of Hail, Dr. Alghamdi is a member of high committee for recruitment and the high committee for projects follow-up. He has undertaken many projects funded by University of Ha'il and other Research Organizations. He published more than 40 journal papers in the field of materials science and nanotechnology. He is honorable editor of international journal and editor in chief of the journal of science at University of Ha'il, and a reviewer of several international journals. 